\title{
EDITORIAL
}

\section{An infectious disease pandemic and increased suicide risk}

Leo Sher $^{1,2}$ iD

${ }^{1}$ James J. Peters Veterans' Administration Medical Center, Bronx, NY, USA. ${ }^{2}$ Department of Psychiatry, Icahn School of Medicine at Mount Sinai, New York, NY, USA.

An outbreak of coronavirus disease 2019 (COVID-19) started in China in December 2019 and has proliferated to other Asian countries, Europe, North and South America, Australia, and Africa. This epidemic may increase suicide risk. A community outbreak of severe acute respiratory syndrome (SARS) in 2003 in Hong Kong was associated with an increased risk of suicide among older people. ${ }^{1}$ There are three factors that may increase suicide risk during an epidemic of an infectious disease: a) anxiety and uncertainty; b) social isolation; and c) economic issues.

The COVID-19 pandemic has caused fear, anxiety, and distress in the general population and, especially, among individuals with psychiatric disorders. Anxiety is fueled by uncertainty. People are struggling with the uncertain nature of this very contagious and potentially deadly disease. A neuroscience study has shown that uncertainty is a more stressful state than really knowing something bad will occur. $^{2}$ Anxiety is also fueled by anxiety-inducing publications in mass media and alarmist posts on social media. In fact, the coronavirus epidemic has monopolized the media. Anxiety, distress, and uncertainty may trigger suicidal ideation and suicide attempts in individuals with psychiatric disorders and in low-resilient individuals without psychiatric diagnoses. ${ }^{3}$ Individuals with severe medical illnesses such as cancer may also become suicidal. When the future is murky with uncertain prospects and results, suicide risk is elevated. Stress and anxiety contributed to the higher suicide rate among older people during the 2003 SARS outbreak in Hong Kong. ${ }^{1}$ Perhaps resilience factors are more important forecasters of the development of suicidality than the extent of exposure to stressful situations such as the COVID-19 epidemic.

Many countries have enacted lockdown measures limiting gatherings and social contact, disrupting the lives of millions of people. The COVID-19 epidemic has left many people more alone than they've been in a long time, or ever. Social isolation negatively affects the psychological well-being of both psychiatric patients and healthy individuals. Individuals with psychiatric illnesses usually need more - not less - social interaction. Many persons with psychiatric disorders live alone and stay locked down for long periods. Loneliness may lead to despair, and social isolation is a suicide risk factor. ${ }^{4}$ Multiple observational

Correspondence: Leo Sher, James J. Peters VA Medical Center, 130 West Kingsbridge Road, Bronx, NY, 10468, USA.

E-mail: Leo.Sher@mssm.edu

Submitted Mar 28 2020, accepted Apr 012020. studies suggest that both objective social isolation (e.g., living alone) and subjective feeling of loneliness are associated with suicidal ideation and suicide attempts. ${ }^{4}$ These findings were consistent across different cultures. ${ }^{4}$ Social disengagement played a role in the increased suicide rate among older adults during the SARS epidemic in Hong Kong in 2003. ${ }^{1}$ One third of SARS-related suicide victims experienced social isolation. ${ }^{1}$

The operations of many thousands of businesses around the world have been suspended during the infectious disease pandemic. Millions of people have temporarily or permanently lost their income. Financial problems are frequently associated with a loss of self-esteem, hopelessness, depression, alcohol and drug abuse, domestic difficulties, and other issues. ${ }^{5}$ It is important to note that lower income reduces access to psychiatric care. The capacity of people with psychiatric disorders to compete in the job market is frequently reduced, i.e., it is difficult for them to find a new job. ${ }^{5}$ Therefore, an economic downturn may lead to suicidal behavior in people with psychiatric disorders and other vulnerable individuals. ${ }^{5}$

A combination of anxiety, uncertainty, social isolation and economic problems may significantly increase suicides. Mental health professionals should stay in touch with their patients to reduce their anxiety and isolation. People need to be advised to connect as best they can through technology. Whenever possible, mental health professionals should advocate for patients who are experiencing financial or other difficulties. Individuals with a history of suicide attempt need follow up during stressful times. Both psychiatric patients and the general public should be advised to limit the use of media, including social media.

It is important to reduce access to lethal means. For example, to decrease firearm suicide death, firearm owners should be advised to store firearms unloaded, separate from ammunition, and in a secure place like a gun safe. Putting time and space between a distressed individual and a firearm may reduce impulsive suicides.

Suicide remains an important public health problem. As health care professionals respond forcefully to an infectious disease pandemic, mental health clinicians should not lose sight of suicide prevention.

How to cite this article: Sher L. An infectious disease pandemic and increased suicide risk. Braz J Psychiatry. 2020;42:239-240. http://dx.doi.org/10.1590/1516-4446-2020-0989 


\section{Disclosure}

The author reports no conflicts of interest.

\section{References}

1 Yip PS, Cheung YT, Chau PH, Law YW. The impact of epidemic outbreak: the case of severe acute respiratory syndrome (SARS) and suicide among older adults in Hong Kong. Crisis. 2010;31:86-92.
2 de Berker AO, Rutledge RB, Mathys C, Marshall L, Cross GF, Dolan $\mathrm{RJ}$, et al. Computations of uncertainty mediate acute stress responses in humans. Nat Commun. 2016;7:10996.

3 Sher L. Resilience as a focus of suicide research and prevention. Acta Psychiatr Scand. 2019;140:169-80.

4 Calati R, Ferrari C, Brittner M, Oasi O, Olié E, Carvalho AF, et al. Suicidal thoughts and behaviors and social isolation: a narrative review of the literature. J Affect Disord. 2019;245:653-67.

5 Mann JJ, Metts AV. The economy and suicide. Crisis. 2017;38: $141-6$. 